\title{
AN INTEGRAL INVOLVING THE GENERALIZED ZETA FUNCTION
}

\author{
E. ELIZALDE and A. ROMEO \\ Dept. of Structure and Constituents of Matter, \\ Faculty of Physics, University of Barcelona, \\ Diagonal 647, 08028 Barcelona.
}

(Received February 21, 1989 and in revised form May 5, 1989)

\begin{abstract}
A general value for $\int_{a}^{b} d t \log \Gamma(t)$, for $a, b$ positive reals, is derived in terms of the Hurwitz $\zeta$ function. That expression is checked for a previously known special integral, and the case where $a$ is a positive integer and $b$ is half an odd integer is considered. The result finds application in calculating the numerical value of the derivative of the Riemann zeta function at the point -1 , a quantity that arises in the evaluation of determinants of Laplacians on compact Riemann surfaces.
\end{abstract}

KeY WORDS AND PHrases. Zeta function, Determinants, Riemann Surfaces, String Theories.

1980 AMS SUBJECT CLASSIFICATION CODES. 33A15, 30B40, 30F30.

\section{Introduction.}

The geometry of the Laplacian operator on compact Riemann surfaces has been object of renewed interest in the context of string theory path integrals for the closed bosonic Polyakov string in the critical dimension $d=26$. In that framework, sums over multiloop amplitudes lead to physical quantities depending on the determinants of these laplacians.

D'Hoker and Phong [1,2] realized that the physically interesting determinants admit relatively simple expressions in terms of Selberg zeta functions. Let $M$ be a Riemann surface with genus $g \geq 2$. If $\Delta_{n}^{ \pm}$are the Laplacians acting on tensors of rank $\pm n$ on this surface, their determinants can be calculated by applying the zeta function 
method, which establishes

$$
\operatorname{Det}^{\prime} \Delta_{n}^{ \pm}=\exp \left(-\zeta_{n}^{ \pm}{ }^{\prime}(0)\right)
$$

where $\zeta_{n}^{ \pm}$are the associated operator zeta functions, the prime on Det means deletion of the zero mode, and the other means derivative with respect to $s$. For the sake of clarity, let us focus on the case $n=0$, corresponding to the Laplacian acting on scalar functions. It turns out that the associated zeta function can be split into two pieces

$$
\zeta_{0}(s)=\zeta_{0}^{(e)}(s)+\zeta_{0}^{(r)}(s)
$$

with

$$
\zeta_{0}^{(e)}(s)=\frac{g-1}{\sqrt{\pi} \Gamma(s)} \int_{0}^{\infty} d b \frac{b^{s-1 / 2}}{\operatorname{sh} b / 2} K_{3 / 2-s}(b / 2) .
$$

On the other hand $M$ can be identified with $H / \Gamma$, the action of some Fuchsian group $\Gamma$ on the upper half-plane $H=\{z=x+i y: y>0\}$ endowed with the Poincare metric $d s^{2}=y^{-2}\left(d x^{2}+d y^{2}\right)$. Going one step further, D'Hoker and Phong [2] have shown that, introducing the Selberg zeta function

$$
Z(s) \equiv \prod_{\gamma \text { prim. }} \prod_{p=0}^{\infty}\left(1-e^{-(s+p) l(\gamma)}\right)
$$

where the first product runs over the interconjugate primitive elements $\gamma$ of $\Gamma$ and $l(\gamma)$ are the lengths of the corresponding geodesics measured with the Poincare metric (see McKean [3]) one gets

$$
\zeta_{0}^{(r)}{ }^{\prime}(0)=-\log Z^{\prime}(1)
$$

That is an elegant result about which we are not going to make any further comment here. We will, instead turn to $\zeta_{0}^{(e)}$. The value given by D'Hoker and Phong [2] is

$$
\zeta_{0}^{(e) '}(0)=(g-1)\left(-\log 2 \pi+\frac{1}{2}-4 \zeta^{\prime}(-1)\right)
$$

which coincides with formula (24a) in [4], $\zeta(s)$ is the Riemann zeta function. Then from (1.1), (1.2) and (1.5),

$$
\operatorname{Det}^{\prime} \Delta_{0}=Z^{\prime}(1) e^{-\zeta_{0}^{(0)}{ }^{\prime}(0)}
$$

The exponent contains $\zeta^{\prime}(-1)$ as a relevant constant, so some understanding of this number and its occurrence in (1.6) would be desirable. While D'Hoker and Phong [2] put the emphasis on the derivation of (1.5), we would like to go over the calculation leading to (1.6) in some detail in the next section, check the degree of non-triviality of that result, and obtain possible mathematical consequences.

In particular, we shall be led to derive an explicit expression (non-existent to our knowledge in the mathematical literature) for the integral of $\log \Gamma(t)$ between any two positive reals, the result being given in Sect. 3. In Sect. 4 it will be restricted to the particular cases that we need explicitly, and in Sect. 5 a series expansion for $\zeta^{\prime}(-1)$ which converges very rapidly will be obtained on the basis of this analysis. 


\section{The role of the Riemann zeta function in $\zeta_{0}^{(e)}$.}

Let us now turn to expression (1.3). $K_{\nu}(x)$ can be put as

$$
K_{\nu}(x)=\frac{\pi}{2 \sin \pi \nu} \sum_{k=0}^{\infty}\left(\frac{(x / 2)^{2 k-\nu}}{k !(k-\nu !)}-\frac{(x / 2)^{2 k+\nu}}{k !(k+\nu !)}\right) .
$$

Then, one can plug (2.1) into (1.3) and take advantage of the integral representation for the Hurwitz generalized zeta function

$$
\zeta(z, q)=\frac{1}{\Gamma(z)} \int_{0}^{\infty} d t \frac{t^{z-1} e^{-t q}}{1-e^{-t}}, \operatorname{Re} z>1, q>0 .
$$

As a result, two formal series show up

$$
\begin{aligned}
\zeta_{0}^{(e)}(s)=\frac{g-1}{\sin (\pi(3 / 2-s))} & {\left[\sum_{k=0}^{\infty} \frac{2^{-k+1} \Gamma(k+s)}{\Gamma(s) \Gamma(k+1)} \zeta(2 k+2 s-1,1 / 2)\right.} \\
& \left.-\sum_{k=0}^{\infty} \frac{2^{-2 k+2 s-2} \Gamma(k+3 / 2)}{\Gamma(s) \Gamma(k+5 / 2-s)} \zeta(2 k+2,1 / 2)\right],
\end{aligned}
$$

where use has been made of the gamma function reduplication formula.

It should be noticed that the terms with $k=0$ and $k=1$ in the first summatory need a bit of care. Taking into account the expansion $\zeta(1+\varepsilon, q)=\frac{1}{\varepsilon}-\psi(q)+0(\varepsilon)$, and evaluating the derivative at $s=0$ one gets

$$
\begin{gathered}
\left.\frac{d \zeta_{0}^{(e)}(s)}{d s}\right|_{s=0}= \\
-(g-1)\left[\left.2 \frac{d}{d s} \zeta(2 s-1,1 / 2)\right|_{s=0}-\frac{1}{2} \psi\left(\frac{1}{2}\right)-2 \sum_{k=2}^{\infty} \frac{(-1)^{k}}{2^{k}} \frac{1}{k+1} \zeta(k, 1 / 2)\right] .
\end{gathered}
$$

Since

$$
\zeta(s, 1 / 2)=\left(2^{s}-1\right) \zeta(s),
$$

the series that has appeared gives rise to expansions of the form $\sum_{n=2}^{\infty}(-1)^{n} \frac{\zeta(n)}{n+1} z^{n}$. Then, one can recall that $[5]$

$$
\log t !=-\gamma t+\sum_{n=2}^{\infty}(-1)^{n} \frac{\zeta(n)}{n} t^{n}, \text { for }-1<t \leq 1,
$$

with $t ! \equiv \Gamma(t+1)$ and $\gamma$ denoting the Euler-Mascheroni constant. So the expansions in question can be interpreted as

$$
\begin{aligned}
\sum_{n=2}^{\infty}(-1)^{n} \frac{\zeta(n)}{n+1} z^{n} & =\frac{1}{z} \int_{0}^{z} d t t \frac{d}{d t} \log t !+\frac{\gamma}{2} z \\
& =\log \Gamma(z+1)-\frac{1}{z} \int_{1}^{z+1} d t \log \Gamma(t)+\frac{\gamma}{2} z,
\end{aligned}
$$

where a partial integration has been performed. The values we need are precisely $z=1$ and $z=1 / 2$. For $z=1$, the result is known, and reads $\int_{1}^{2} d t \log \Gamma(t)=-1+1 / 2 \log 2 \pi$. Differentiating (2.5) and bearing in mind the identity $\psi(1 / 2)=-\gamma-2 \log 2$, one can reach the intermediate result

$$
\begin{aligned}
\zeta_{0}^{(e) '}(0)=-(g-1) \quad\left[-2 \zeta^{\prime}(-1)+\right. & (2 \zeta(-1)-2) \log 2-2+2 \log 2 \pi \\
& \left.-4 \int_{1}^{3 / 2} d t \log \Gamma(t)\right],
\end{aligned}
$$


where the integral comes from the series corresponding to $z=1 / 2$. As the expression stands, this is the only unknown term.

In addition, an integral like this one was considered by Steiner [4] in connection with the evaluation of the Selberg zeta function for a Laplace operator acting on scalars on a Riemann surface. There the result was given with the aid of the Barnes $G$ function.

A second observation is the following. Had we taken $\Delta_{n}^{ \pm}$instead of $\Delta_{0}$ (see[2]), after carrying out a similar calculation we would have ended up with $\int_{1}^{n+3 / 2} d t \log \Gamma(t)$.

\section{INTEGRATION OF $\log \Gamma(t)$.}

We will follow a quite simple procedure based on two formulæ for partial derivatives of the generalized zeta function given by (2.2), which can also be defined as

$$
\zeta(s, t)=\sum_{k=0}^{\infty} \frac{1}{(k+t)^{s}}, \text { for } \operatorname{Re} s>1, t \neq 0,-1,-2, \ldots
$$

In addition, it admits an analytic continuation that makes it regular for every $s$, except for $s=1$, where it has a simple pole. Derivation of (3.1) with respect to $t$ gives.

$$
\frac{\partial}{\partial t} \zeta(s, t)=-s \zeta(s+1, t)
$$

On the other hand, it has been long known (e.g. Erdélyi et al. [5], pg. 26) that the partial derivative of the Hurwitz zeta function with respect to $s$ at $s=0$ is related to Euler's gamma function by

$$
\left.\frac{\partial}{\partial s} \zeta(s, t)\right|_{s=0}=\log \Gamma(t)-\frac{1}{2} \log 2 \pi, \operatorname{Re} t>0 .
$$

By partial derivation of (3.2) with respect to $s$, one is led to

$$
\frac{\partial^{2}}{\partial s \partial t} \zeta(s, t)=-\zeta(s+1, t)-s \frac{\partial}{\partial s} \zeta(s+1, t),
$$

and, after applying (3.2) again, to

$$
\frac{\partial^{2}}{\partial s \partial t} \zeta(s, t)=-\frac{1}{s} \frac{\partial}{\partial t} \zeta(s, t)-s \frac{\partial}{\partial s} \zeta(s+1, t),
$$

which is valid for $s \neq 0,1$. At this point, it is important to notice that for $s \neq 1$, and $\operatorname{Re} t>0, \zeta(s, t)$ is an analytic function of $s$ and $t$, and therefore, the partial derivatives with respect to $s$ and to $t$ commute in this region. Besides, by taking $s=-1$ and making use of (3.3), we obtain

$$
\left.\frac{\partial^{2}}{\partial t \partial s} \zeta(s, t)\right|_{s=-1}=\left.\frac{\partial}{\partial t} \zeta(s, t)\right|_{s=-1}+\log \Gamma(t)-\frac{1}{2} \log 2 \pi .
$$

When integrating the above expression with respect to $t$ over a positive real interval $(a, b)$, we get

$$
\int_{a}^{b} d t \log \Gamma(t)=\left[\zeta(-1, t)+\zeta^{\prime}(-1, t)+\frac{t}{2} \log 2 \pi\right]_{a}^{b}
$$


where $\zeta^{\prime}(-1, t)$ denotes $\left.\frac{\partial}{\partial t} \zeta(s, t)\right|_{s=-1}$.

Eq. (3.7) is the desired general formula and one of the main results in this paper. Although, it is valid for $a, b>0$, we feel that it can be extended to any pair of negative reals provided that there is no singularity of the $\Gamma$ function between them, i.e. to any interval containing neither the origin nor negative integers. In the usual integral handbooks (see e.g. Gröbner and Hofreiter [6]) only the special case $b=a+n, n \in \mathbb{N}$ appears. One can also find a more general result based on the Barnes $G$ function, which generalizes Euler's gamma function (see Steiner [4] and references therein) but, to our knowledge, no result in terms of $\zeta$ hảs been listed.

As a way of testing (3.7), let us check a previously known case, whose result can be found in several places (e.g. Erdélyi et al. [5] pg. 24), namely,

$$
\int_{a}^{a+n} d t \log \Gamma(t)=\sum_{k=0}^{n-1}(a+k) \log (a+k)-n a-\frac{n(n-1)}{2}+\frac{n}{2} \log 2 \pi, a \in \mathbf{R}, n \in \mathbf{N}^{*} .
$$

When evaluating this particular integral according to (3.7), we get the differences $\zeta(-1, a+n)-\zeta(-1, a)$ and $\zeta^{\prime}(-1, a+n)-\zeta^{\prime}(-1, a)$. For our purpose, we can take advantage of the identity

$$
\zeta(s, a+n)=\zeta(s, a)-\sum_{k=0}^{n-1}(k+a)^{-s},
$$

trivially obtained from (3.1). The point of this expression is that, while (3.1) was valid just for $s>1$, the second term on the r.h.s. of (3.9) is finite for any value of $s$. Besides, both $\zeta(s, a+n)$ and $\zeta(s, a)$ admit analytic continuations to $s<1$. Therefore (3.9) makes sense for $s<1$ as well. The same reasoning applies to its derivative with respect to $s$ :

$$
\zeta^{\prime}(s, a+n)=\zeta^{\prime}(s, a)+\sum_{k=0}^{n-1}(k+a) \log (k+a), \text { for } s \neq 1 .
$$

Since both (3.9) and (3.10) hold for any $s \neq 1$, they can be taken at $s=-1$, where they turn out to be

$$
\begin{gathered}
\zeta(-1, a+n)=\zeta(-1, a)-\sum_{k=0}^{n-1}(k+a), \\
\zeta^{\prime}(-1, a+n)=\zeta^{\prime}(-1, a)+\sum_{k=0}^{n-1}(k+a) \log (k+a) .
\end{gathered}
$$

By taking (3.7) for $b=a+n$ and plugging the above identities into the r.h.s., we immediately see that the result is the same as the one given by (3.8) .

\section{Particular cases.}

We are interested in the case where the lower limit of integration is a positive integer and the upper limit is half an odd integer, i.e.

$$
I(m, n) \equiv \int_{m}^{m+n+1 / 2} d t \log \Gamma(t), \quad m \in \mathbf{N}, n \in \mathbf{N}^{*} .
$$


A possible way of calculating this would be to replace $a$ with $m$ and $b$ with $m+n+1 / 2$ on the r.h.s. of (3.7). However, there is an alternative method which shows that all the integrals of the type (4.1) reduce to known quantities plus $I(1,0)$, i.e. plus an integral from 1 to $3 / 2$.

By splitting the integration region into two pieces,

$$
I(m, n)=\int_{m}^{m+n} d t \log \Gamma(t)+\int_{m+n}^{m+n+1 / 2} d t \log \Gamma(t)
$$

the first intergal can be read from (3.8). As for the second one, it is convenient to make the obvious variable change $u=t-m-n+1$ and take advantage of the simple relation

$$
\log \Gamma(u+m+n-1)=\sum_{k=0}^{m+n-2} \log (u+k)+\log \Gamma(u) .
$$

Then, the terms in the summatory can be immediately integrated. As a result of all this, (4.1) becomes

$$
\begin{aligned}
I(m, n)= & -\sum_{k=1}^{m-1} k \log k+\frac{1}{2} \sum_{k=1}^{m+n-1}(2 k+1) \log (2 k+1) \\
& -m n-\frac{n(n-1)}{2}-\frac{1}{2}(m+n-1)-\frac{1}{2}(m+n)^{2} \log 2-\frac{1}{2} \\
& +\frac{n}{2} \log 2 \pi+\int_{1}^{3 / 2} d u \log \Gamma(u) .
\end{aligned}
$$

So, the question of knowing the value of $I(m, n)$ boils down to calculating the integral from 1 to $3 / 2$, that will be now quickly evaluated. (3.7) tells us that

$$
\int_{1}^{3 / 2} d t \log \Gamma(t)=\zeta(-1,3 / 2)-\zeta(-1)+\zeta^{\prime}(-1,3 / 2)-\zeta^{\prime}(-1)+\frac{1}{4} \log 2 \pi
$$

where $\zeta(s, 1)=\zeta(s)$, being $\zeta(s)$ the Riemann zeta function.

From (3.9) and the relation (2.5) it is plain that

$$
\zeta(s, 3 / 2)=\left(2^{s}-1\right) \zeta(s)-2^{s} .
$$

Derivation of this expression with respect to $s$ yields

$$
\zeta^{\prime}(s, 3 / 2)=\left(2^{s} \log 2\right) \zeta(s)+\left(2^{s}-1\right) \zeta^{\prime}(s)-2^{s} \log 2 .
$$

Once again, by analytic continuation (4.6) and (4.7) are valid for any $s \neq 1$. In particular, they can be taken at $s=-1$, thus obtaining

$$
\zeta\left(-1, \frac{3}{2}\right)=-\frac{1}{2} \zeta(-1)-\frac{1}{2}
$$

and

$$
\zeta^{\prime}\left(-1, \frac{3}{2}\right)=\frac{1}{2}(\log 2) \zeta(-1)-\frac{1}{2} \zeta^{\prime}(-1)-\frac{1}{2} \log 2 .
$$

Plugging them into (4.5), we get

$$
\begin{aligned}
\int_{1}^{3 / 2} d t \log \Gamma(t)= & -\frac{3}{2} \zeta^{\prime}(-1)+\left(-\frac{3}{2}+\frac{1}{2} \log 2\right) \zeta(-1) \\
& -\frac{1}{2}(1+\log 2)+\frac{1}{4} \log 2 \pi
\end{aligned}
$$


which allows us to write (4.4) as

$$
\begin{aligned}
\int_{m}^{m+n+1 / 2} d t \log \Gamma(t)= & -\sum_{k=1}^{m-1} k \log k+\frac{1}{2} \sum_{k=1}^{m+n-1}(2 k+1) \log (2 k+1) \\
& -\frac{1}{2}(m+n)^{2}(1+\log 2)+\frac{m(m-1)}{2} \\
& -\frac{3}{2} \zeta^{\prime}(-1)+\left(-\frac{3}{2}+\frac{1}{2} \log 2\right) \zeta(-1) \\
& +\frac{n+1 / 2}{2} \log 2 \pi .
\end{aligned}
$$

The Riemann zeta function at the point -1 is well known,

$$
\zeta(-1)=-\frac{B_{2}}{2}=-\frac{1}{12}
$$

( $B_{2}=1 / 6$ being the second Bernoulli number), but the remarkable feature of expression (4.11) is the fact that $\zeta^{\prime}(-1)$ appears as a relevant mathematical constant for the calculation of this family of integrals.

These results, combined with (2.8), allow us to recover (1.6).

\section{Calculation of $\zeta^{\prime}(-1)$.}

A numerical value for $\zeta^{\prime}(-1)$ was given by Steiner [4], pg. 452, namely $\zeta^{\prime}(-1) \simeq$ -0.1655 . It was obtained by taking a finite number of terms of the asymptotic expansion for $\zeta^{\prime}(-1, q)$ given by Elizalde in [7] (see also Fried [8]). Nevertheless, in the case $q=1$ the validity of that approximation seems to be, at first sight, difficult to justify. Here we will find the value of $\zeta^{\prime}(-1)$ by means of using the preceding results.

There is at least one alternative way of evaluating the integral on the l.h.s. of (4.10). After doing a trivial variable change, making use of the power series (2.6) and integrating term by term, we get

$$
\int_{1}^{3 / 2} d t \log \Gamma(t)=-\frac{\gamma}{8}+\sum_{n=2}^{\infty} \frac{(-1)^{n}}{2^{n+1} n(n+1)} \zeta(n) .
$$

Using (4.12), a comparison of (4.10) with (5.1) leads to

$$
\begin{aligned}
\zeta^{\prime}(-1)= & -\frac{1}{4}-\frac{7}{36} \log 2+\frac{1}{6} \log \pi+\frac{\gamma}{12} \\
& -\frac{2}{3} \sum_{n=2}^{\infty} \frac{(-1)^{n}}{2^{n+1} n(n+1)} \zeta(n),
\end{aligned}
$$

which provides us with an expression to evaluate $\zeta^{\prime}(-1)$, since the r.h.s. can be numerically calculated. Eq. (5.2) is another main result in this paper. For the even- $n$ terms, we have applied the relation

$$
\zeta(2 m)=(-1)^{m+1} \frac{(2 \pi)^{2 m}}{2(2 m) !} B_{2 m}, m \in \mathbf{N}^{*},
$$

where $B_{2 m}$ is the $2 m^{\text {th }}$ Bernoulli number. As for the odd-n terms, we have used the definition of the Riemann zeta function,

$$
\zeta(n)=\sum_{k=1}^{\infty} \frac{1}{k^{n}}, n>1,
$$


and approximated $\zeta(n)$ with finite sums up to the desired accuracy. The outcome of doing all that is

$$
\zeta^{\prime}(-1)=-0.16542115 \ldots
$$

in good agreement with the value given by Steiner [4]. Notice, moreover, the rapid convergence of the series in (5.2), which can be numerically checked. In fact, using a simple Pascal program we have got the stability of the first six figures of (5.5) after summation of only eleven terms of the series in (5.2).

\section{ACKNOWLEDEGEMENTS}

Thanks are given to the referee of a previous version of this paper for useful advice. This work has been partially supported by the CAICYT, research project AE87-0016-3. One of us (A. R.) has also obtained financial help through an FPPI grant, Programa Areas de Conocimiento, from MEC Spain, during the last stage of this work.

\section{REFERENCES}

[1] D'Hoker, E. and Phong, D.H., On Determinants of Laplacians on Riemann Surfaces, Commun. Math. Phys. 104 (1986) 537-545.

[2] D'Hoker, E. and Phong, D.H., Multiloop Amplitudes for the Bosonic Polyakov String, Nucl. Phys. B 269 (1986) 204-234.

[3] McKean, H.P., Selberg's Trace Formula as Applied to a Compact Riemann Surface, Commun. Pure Appl. Math., 25 (1972) 225-246.

[4] Steiner, F., On Selberg's zeta function for compact Riemann surfaces, Phys. Lett. B 188 (1987) 447-454.

[5] Erdélyi, A., Magnus, W., Oberhettinger,F., and Tricomi, F.G., Higher Transcendental Functions, Mc Graw-Hill, New York, 1953.

[6] Gröbner, W., and Hofreiter, N., Integraltaffel, Zweiter Teil, Springer Verlag, Wien, 1973.

[7] Elizalde, E., Derivative of the generalised Riemann zeta function $\zeta(z, q)$ at $z=-1$, J.Phys. A 18 (1985) 1637-1640, and An asymptotic expansion for the first derivative of the generalized Riemann zeta function, Math. of Computation, 47, 175 (1986) $347-350$.

[8] Fried, D., Analytic torsion and closed geodesics on hyperbolic manifolds, Invent. Math. 84 (1984) 523-540. 


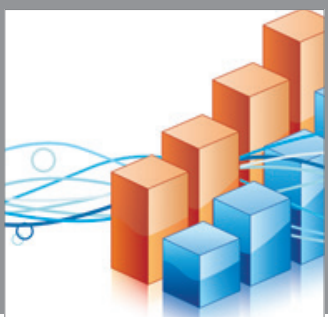

Advances in

Operations Research

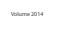

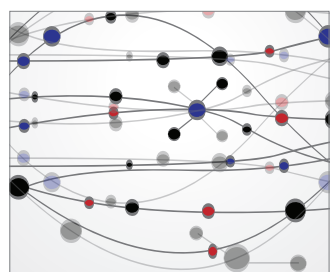

\section{The Scientific} World Journal
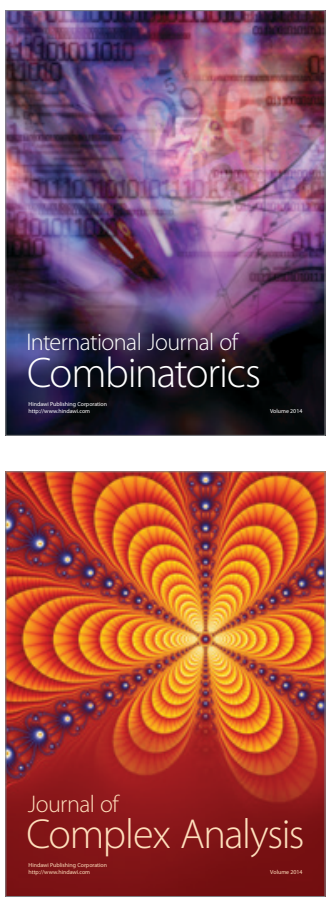

International Journal of

Mathematics and

Mathematical

Sciences
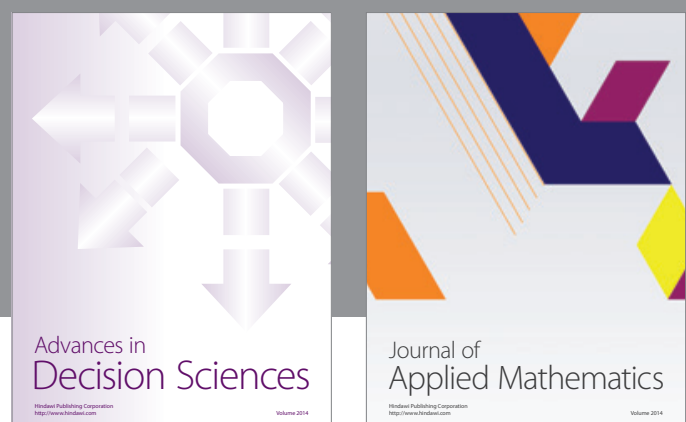

Journal of

Applied Mathematics
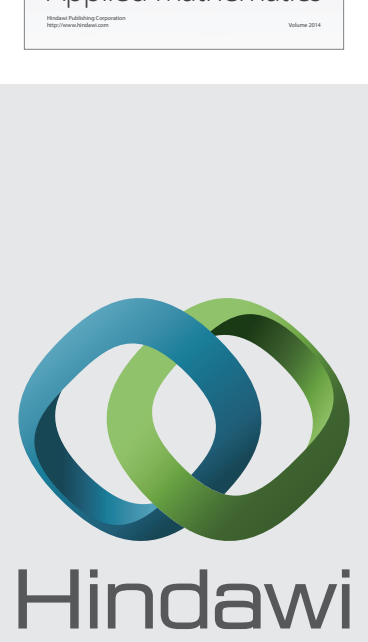

Submit your manuscripts at http://www.hindawi.com
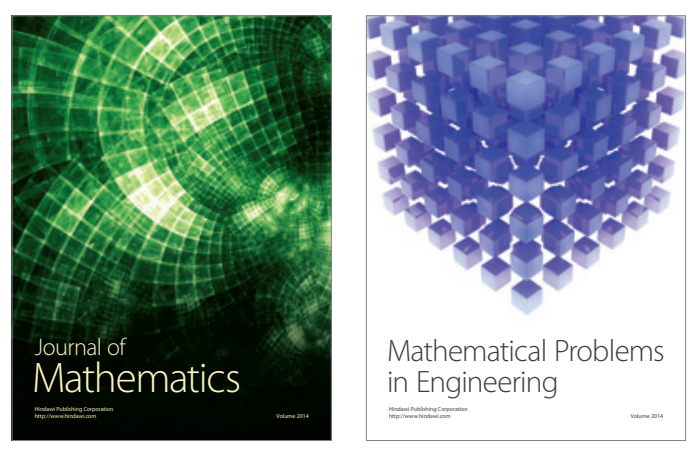

Mathematical Problems in Engineering
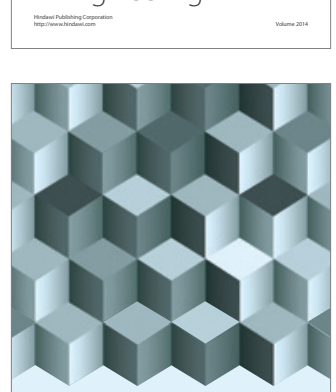

Journal of

Function Spaces
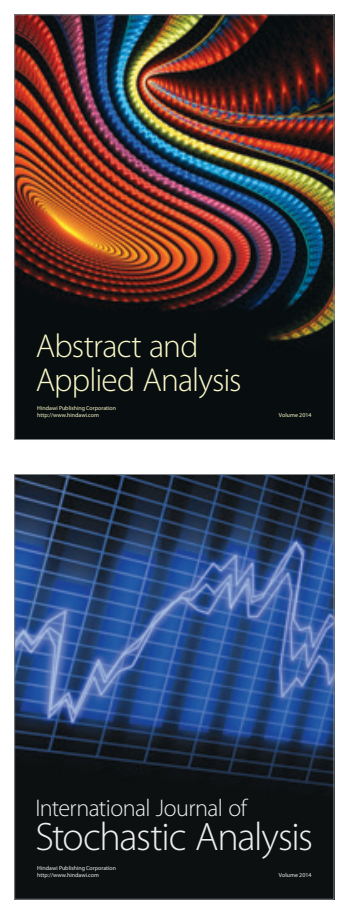

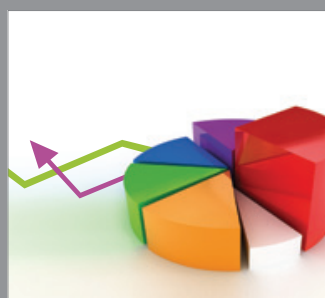

ournal of

Probability and Statistics

Promensencen
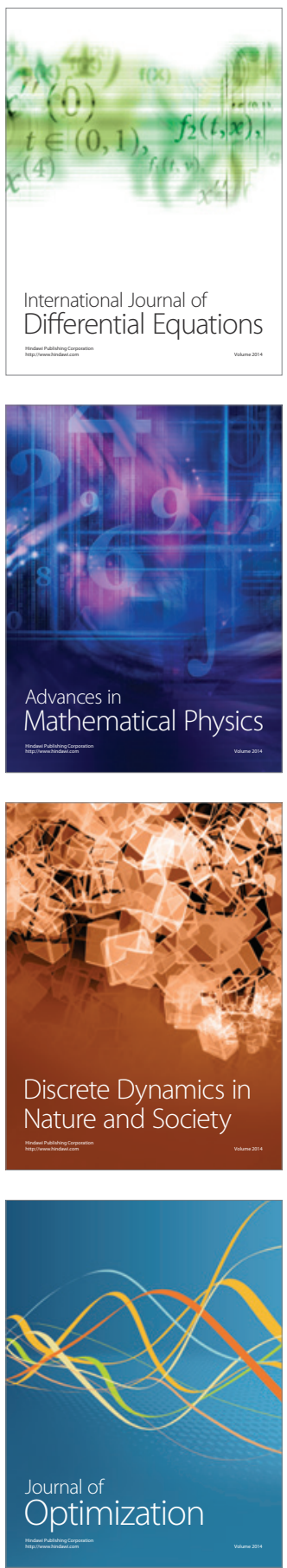